Ingeborga BESZTERDA

\& Beata SZPINGIER

Università Adam Mickiewicz di Poznań

\title{
IL CONTATTO INTERLINGUISTICO : TRA BILINGUISMO E DIGLOSSIA IN ITALIA
}

Il contatto fra lingue e dialetti rappresenta un caso particolare del fenomeno che va sotto il nome di contatto fra lingue. Secondo WEINREICH (1964:1) « due o più lingue si diranno in contatto, se sono usate alternativamente dalle medesime persone ». Va notato che il contatto interlinguistico, avendo luogo tra lingue (o dialetti o sottosistemi) più o meno diverse, può originare forme di deviazione dalle norme di una o di entrambe le lingue, ossia delle interferenze. La descrizione delle interferenze viene fatta di solito in termini di linguistica (ovvero psicolinguistica) contrastiva dal punto di vista fonico, grammaticale e lessicale. Tuttavia, TITONE (1973: 3) osserva che una spiegazione delle interferenze in una situazione di contatto linguistico, includente $\mathrm{i}$ fenomeni di diffusione, persistenza ed evanescenza di un particolare caso interferenziale, è possibile soltanto se si tenga conto di fattori extra-linguistici. Tali fattori extra-linguistici possono essere sia di natura individuale (come l'abilità verbale, la perfezione d'uso delle due lingue, gli atteggiamenti verso ciascuna lingua, e così via, caratteristici di un parlante bilingue e presenti nello stesso in gradi e sfumature diversi), sia di ordine sociale (come la dimensione del gruppo bilingue e la sua omogeneità o differenziazione socio-culturale, atteggiamenti stereotipi verso le due lingue, atteggiamenti verso la cultura di ciascuna comunità linguistica, tolleranza o purismo nell'uso linguistico, ecc.). Lo studioso afferma che per determinare fenomeni di interferenza bisogna rifarsi a un approccio interdisciplinare (linguistica, psicolinguistica e sociolinguistica) e non a una semplice analisi contrastiva a priori delle caratteristiche strutturali dei due sistemi a contatto. Occorre aggiungere inoltre che il contatto interlinguistico rappresenta sostanzialmente un contatto di culture, da qui la preminenza dell'approccio sociolinguistico nell'esame dei fenomeni di bilinguismo e/o diglossia.

Per delineare le diverse situazioni linguistiche presenti oggi in Italia, che riflettono in modo approssimativo gli stadi successivi del trapasso dal monolinguismo dialettale a quello dell'italiano, MIONI (1979 : 108) propone infatti di ricorrere ai concetti sociolinguistici di bilinguismo sociale e di diglossia. Il bilinguismo sociale coincide con la situazione in cui la maggioranza della popolazione padroneggia due lingue diverse, sia passivamente che attivamente. TITONE (1973 : 2) puntualizza : «il bilinguismo è sociale o collettivo quando un intero gruppo (famiglia, comunità, nazione) si vale di due lingue in maniera alternativa ». Con il termine diglossia si indica una situazione in cui esistono due varietà distinte presenti in una comunità, una delle quali è riservata solo a occasioni formali e pubbliche mentre l'altra viene impiegata da tutti in circostanze normali e quotidiane. HUDSON (1996: 56) ricorda che le due varietà vengono chiamate di norma alta (A) e bassa (B), o anche standard e vernacolo. In via preliminare possiamo asserire che riguardo alla realtà italiana queste varietà sarebbero rispettivamente lingua e dialetto. A sostegno della nostra opinione 
occorre menzionare che GUMPERZ (in : TITONE 1973 : 4) ha precisato a più riprese che il fenomeno della diglossia riguarda non soltanto le lingue standardizzate e di grande uso tradizionale, ma anche $\mathrm{i}$ dialetti, registri o varietà funzionalmente differenziate di qualsiasi genere.

Prima di passare ai particolari relativi alla situazione italiana, ricordiamo seguendo TITONE (1973: 6) che i fenomeni di bilinguismo e diglossia sono presenti in vaste e complesse comunità linguistiche, in cui i membri hanno a disposizione sia una gamma di ruoli settorializzati sia un facile accesso a tali ruoli. Per questo motivo si nota la tendenza a conservare vive entrambe le lingue per la loro sufficientemente vasta vitalità e autonomia. Data la eterogeneità sociale di molte attuali comunità (per es. dovuta a fenomeni di immigrazione), è in aumento il numero di comunità caratterizzate da bilinguismo unito a diglossia, il che porta a situazioni di passaggio dal monolinguismo domestico al bilinguismo culturale. Siccome tale schema del contatto linguistico può essere valido altresì per i sottosistemi - come dialetti, registri, varietà funzionali, e così via - ci permettiamo di sottoporre la situazione italiana a un' accurata analisi da questo punto di vista.

La varia combinazione della presenza o assenza dei due fenomeni, con un'eventuale aggiunta di situazioni di monolinguismo, consente di individuare le seguenti definizioni riferite alla realtà italiana (MIONI, 1979 : 109) :

1. monolinguismo dialettale : una realtà del genere era diffusa in Italia nei secoli passati mentre nell'Italia contemporanea non si riscontrano comunità in cui l'italiano non faccia parte del repertorio linguistico almeno a livello passivo ;

2. diglossia senza bilinguismo sociale: tale situazione si riscontrava in passato, mentre nell'Italia di oggi è limitata ad alcune zone rurali e montane e, in particolare, alle zone meno urbanizzate del Sud. Ad avviso di MiONI (ibid.), date le funzioni dell'italiano, padroneggiato solo dalla classe dirigente, non vi è bilinguismo sociale bensì sarebbe più opportuno parlare di bilinguismo alto-borghese ;

3. diglossia con bilinguismo sociale : siccome la maggioranza degli italiani conosce bene sia il dialetto che la lingua (almeno in modo passivo) e condivide le norme che regolano l'uso di una varietà dell'italiano o del dialetto, secondo MIONI questa sarebbe la situazione italiana media che si lascia osservare nelle regioni di sviluppo medio (non toccate dal fenomeno dell'immigrazione oltre la soglia della non-integrazione dei nuovi arrivati) ;

4. bilinguismo sociale senza diglossia: la maggioranza della popolazione padroneggia sia l'italiano che il dialetto, ma la presenza di una notevole percentuale di immigrati provenienti da altre zone rende impossibile l'integrazione dei dialetti per l'uso di tutta la comunità. Le condizioni di scarsa omogeneità dialettale si riscontrano in tutte le zone toccate dal fenomeno di un'immigrazione massiccia (come il triangolo industriale Genova-Torino-Milano, Roma, ecc.). MiONI (1979: 110) osserva che in queste zone i dialetti indigeni vengono usati per 'scopi snobistici' e come forma di discriminazione verso gli immigrati, in particolare i meridionali (mentre questi ultimi ricorrono al dialetto per criptolalìa) ; 
5. monolinguismo standard : in passato caratterizzava tutta l'area dei dialetti toscani con la tendenza a estendersi su vaste aree di Lazio, Umbria e Marche (i cui dialetti non differiscono molto dal toscano), mentre attualmente tende a diffondersi presso le classi medio-alte di tutta l'Italia ${ }^{31}$.

Fatta questa premessa ci proponiamo di passare in rassegna le considerazioni di altri studiosi che cercando di diagnosticare la presente situazione linguistica italiana ne forniscono ciascuno un punto di vista particolare.

La relazione funzionale e di status che intercorre tra l'italiano e il dialetto viene definita da alcuni studiosi (BERRUTO, 2003 : 28) come quella di diglossia, cioè la distribuzione del repertorio fra una varietà linguistica alta, per gli usi scritti e formali, e una varietà linguistica bassa, per gli usi parlati informali.

Anche FREDDI (1983 : 233) accenna a una situazione di diglossia nei rapporti lingua-dialetto osservando che l'italiano è riservato ai rapporti ufficiali, formali e ai domini elevati, mentre il dialetto è destinato ai rapporti informali e immediati della famiglia, del ristretto gruppo di amici o del paese.

TRUMPER (1977 : 260) tenta di affinare la suddetta distinzione introducendo i termini macrodiglossia e microdiglossia per dar rilievo alle differenze tra aree (o anche classi sociali) in cui il dialetto è assai più debole. Ricordiamo brevemente $\mathrm{i}$ tratti particolari di questa distinzione (con riferimento alla situazione italiana) presentati da BERRUTO (2005 : 199).

\begin{tabular}{|l|l|}
\hline \multicolumn{1}{|c|}{ Macrodiglossia } & \multicolumn{1}{c|}{ Microdiglossia } \\
\hline $\begin{array}{l}\text { - entrambi i codici sono distribuiti su un } \\
\text { ampio numero di domini }\end{array}$ & $\begin{array}{l}\text { - un codice è usato in pochissimi } \\
\text { domini }\end{array}$ \\
\hline - formazione di una koinè dialettale & - assenza di koinè dialettale \\
\hline $\begin{array}{l}\text { - ampia sovrapposizione fra i codici in } \\
\text { contesti funzionalmente ambigui }\end{array}$ & $\begin{array}{l}\text { - netta separazione funzionale fra i due } \\
\text { codici }\end{array}$ \\
\hline $\begin{array}{l}\text { - enunciati mistilingui comuni } \\
\text { nell'interazione quotidiana }\end{array}$ & \begin{tabular}{l} 
- le varietà di solito non si mescolano \\
\hline - i dialetti sono socialmente stratificati
\end{tabular} \\
\hline
\end{tabular}

Come si può vedere, il criterio fondamentale a cui ricorre TRUMPER (1977 : 260) poggia sulla forza della varietà $\mathrm{B}$, il dialetto. Nella condizione di macrodiglossia, il dialetto è, dal punto di vista sociolinguistico, abbastanza vitale : si suddivide in varietà interne (ad es. dialetti locali, cioè vernacoli) e converge con la lingua (varietà $\mathrm{A}$ ) in molti ambiti, il che porta ai frequenti casi di commutazione

\footnotetext{
${ }^{31}$ Dalle recenti indagini DoXA (in : COVERI, 1998) risulta che circa il 25\% degli intervistati dichiara di conoscere solo l'italiano e il $7 \%$ solo il dialetto.
} 
di codice $^{32}$ o alla produzione di enunciati mistilingui, quando « italiano e dialetto si fronteggino direttamente » (BERRUTO, 1999: 31). La macrodiglossia caratterizza in particolare le regioni come il Veneto e la Campania, mentre secondo Mioni (1979: 109) sarebbe valida anche per il Piemonte centrosettentrionale e probabilmente per la Sicilia. Nella situazione di microdiglossia, al contrario, il dialetto è sociolinguisticamente assai debole, essendo confinato a pochi domini d'uso, ed è tenuto ben separato dalla lingua. Il fenomeno in questione sarebbe tipico dell'Emilia-Romagna e del Piemonte; MIONI (1977 : 109) aggiunge inoltre le Marche e la Calabria. Lo stesso MIONI (1979: 109) paragona questa situazione alle condizioni di patois in Francia, dove il dialetto è usato solo nell'ambito di un villaggio nelle comunicazioni tra familiari e amici stretti.

Per BERRUTO (1977 : 17) la situazione normale della popolazione italiana è ancora una situazione di bilinguismo lingua nazionale (in una sua varietà regionale) - dialetto, con un'estrema varietà del comportamento nell'intervallo tra le due estremità : $\mathrm{i}$ parlanti in genere hanno a disposizione alcune varietà intermedie e oscillano - in relazione a fattori socio-culturali come l'età, la classe sociale, il grado di istruzione, ecc. - fra un massimo di competenza e di uso del solo italiano, con conoscenza passiva del dialetto locale, e un massimo di competenza e uso del solo dialetto, con conoscenza poco più che passiva dell'italiano. Quanto invece al processo di italianizzazione dei dialettofoni, l'italiano costituisce un nuovo codice linguistico (la cosiddetta lingua seconda) ${ }^{33}$, perciò « sembra legittimo parlare di diglossia ».

Secondo FRANCESCATO (1978: 127), nonostante sia il bilinguismo sia la diglossia possano caratterizzarsi nei termini della capacità di ricorrere a linguaggi diversi (o varietà diverse dello stesso linguaggio) da parte dello stesso parlante, occorre rilevare che tanto per il bilinguismo quanto per la diglossia la selezione di tali varietà «non è abbandonata al caso o alla momentanea inclinazione » bensì è sottoposta di regola a criteri ben precisi (abitualmente non espliciti) che possono essere tutti riassunti nell'unico criterio fondamentale del linguaggio più opportuno. Lo studioso osserva pertanto che il parlante italiano nella maggior parte dei casi è incapace di scegliere in maniera adeguata un certo livello linguistico che « pure dovrebbe fare parte, almeno approssimativamente, del suo repertorio », o addirittura «non riesce neppure a estendere il suo repertorio fino a tale livello»(FRANCESCATO, 1978: 128). Questa diagnosi severa porta ad avanzare un'ipotesi sulla scarsa competenza comunicativa dei parlanti italiani (su questo argomento ritorneremo in seguito, quando affronteremo il problema del rapporto lingua/dialetto nell'insegnamento $)^{34}$. Nella realtà sociolinguistica italiana quindi, pur essendoci strumenti linguistici «duttili ed efficienti » perfino sul piano elevato, il corretto impiego della lingua maggiore resta « precluso a una parte non

\footnotetext{
${ }^{32} \mathrm{Si}$ tratta « dell'uso alternato di (varietà di) italiano e (varietà di) dialetto nel corso dello stesso evento comunicativo da parte dello stesso parlante, addirittura all'interno della stessa battuta o frase $»$ (BERRUTO, 1999 : 31).

${ }_{33}^{33}$ Lo confermano, tra gli altri, anche FREDDI (1983) e FrANCESCATO (1978).

34 «La competenza comunicativa permette di scegliere il modo più appropriato per trasmettere un'informazione $\mathrm{o}$ formulare una richiesta o un rimprovero, in rapporto alle circostanze dell'emissione : ci fa scegliere il registro appropriato, la terminologia più adatta all'argomento $[\ldots]$ » (MIONI, 1979: 101).
} 
trascurabile della popolazione ${ }^{35}$. In base a queste osservazioni, FRANCESCATO (1978 : 128) conclude affermando che il problema della diglossia in Italia «si rivela assai più complesso e difficile della semplice opposizione tra la lingua alta e bassa suggerita da FERGUSON ». Così, osserva FRANCESCATO (1978 : 145), per quanto riguarda l'idea della diglossia bisogna guardarsi da non trarre conclusioni affrettate che da una parte portano a intendere lingua come linguaggio alto e dialetto come linguaggio basso, e dall'altra, rifacendosi ai codici di Berenstein inducono a una facile identificazione della lingua col codice elaborato, e il dialetto con quello ristretto : « identificazione semplicistica e contro la quale conviene stare in guardia » (1978: 135); oppure continuando su questa strada, indicare nell'opposizione in parola una differenza di classi sociali, o almeno di status socioeconomico $($ lingua $=$ classi privilegiate, dialetto $=$ classi non privilegiate, $\mathrm{o}$ addirittura lingua $=$ classi egemoni, dialetto = classi subalterne $)$. Così, secondo lo studioso, la semplice opposizione italiano/dialetto « è insufficiente per definire una realtà caratterizzata da altri fattori linguistici, quali italiano regionale, popolare e i dialetti regionali » (ibid.).

Infatti conferma BERRUTO (1999: 4) che nonostante l'uso nel parlato quotidiano tanto della varietà bassa (il dialetto) quanto della varietà alta (l'italiano), l'assetto linguistico italiano non coincide perfettamente con i requisiti di diglossia indicati da FERGUSON (1964 : 429) ${ }^{36}$. BERRUTO (1999 : 5) suggerisce di definire il repertorio italo-romanzo medio in termini di bilinguismo endogeno a bassa distanza strutturale con dilalia. Mediante questo concetto vengono designati dei casi in cui si nota l'uso e la compresenza di due diversi (dia)sistemi linguistici, la cui differenza strutturale ( $s i$ tratta di varietà romanze dello stesso ceppo e sottoposte all'influsso livellatore della lingua standard) è inferiore a quella riscontrabile nei repertori bilingui classici. Tale bilinguismo è di natura interna al sistema linguistico (endogeno) e non deriva da migrazioni di popolazioni più o meno recenti. BERRUTO (1999: 6) mentre caratterizza il rapporto funzionale e di status tra la varietà alta e quella bassa ricorre alla nozione della dilalia « vale a dire, con entrambe le varietà impiegate / impiegabili nella conversazione quotidiana e con uno spazio relativamente ampio di sovrapposizione ». Il concetto di dilalia si oppone direttamente a quello di diglossia ed è stato introdotto in seguito al riconoscimento della scarsa pertinenza del concetto di diglossia in relazione al rapporto fra italiano e dialetti in Italia, che sembra oggi chiaramente diversa rispetto alle comunità classicamente diglottiche, in particolare riguardo a uno dei punti cardinali propri della diglossia, cioè l'assenza di A nell'uso parlato conversazionale comune.

Si noti che si possono individuare (BERRUTO, 2005 : 205) quattro tipi diversi di rapporti sociolinguistici fra le lingue e le varietà di lingua compresenti in un repertorio : bilinguismo sociale (o comunitario, o collettivo), diglossia, dilalia e bidialettismo (o polidialettismo). Dato che tutti e quattro i casi presentano una

\footnotetext{
${ }^{35}$ Con il concetto di lingua maggiore si designa la lingua standard di una comunità di alto livello culturale (FERGUSON, 1971, in : FRANCESCATO, $1978: 128$ ).

${ }^{36}$ BERRUTO (2005: 193) rileva che la sostanziale differenza sta nel fatto che nella situazione di diglossia nessun segmento della comunità parlante usa $\mathrm{A}$ per la normale conversazione quotidiana, e anzi, l'eventuale uso di A in questa viene sentito come pedante e artificiale, in certi casi anche sleale nei confronti della comunità.
} 
certa attinenza (pertanto in misura diversa) alle condizioni linguistiche italiane ci permettiamo di caratterizzare brevemente i modelli evocati.

Si parlerà di bilinguismo sociale o comunitario nella situazione in cui due lingue nettamente diverse ed entrambe elaborate (quindi due lingue di cultura) possono essere impiegate, senza subordinazione funzionale, sia negli usi scritti formali che nella conversazione informale: ad es. la comunità parlante di Montréal (Canada) con bilinguismo sociale francese-inglese ; la Valle d'Aosta, con bilinguismo sociale italiano-francese.

Nella diglossia, i due codici compresenti nella comunità possono essere tanto varietà nettamente divergenti e autonome a livello di struttura e storia della stessa lingua (così anche nella dilalia), quanto lingue diverse, in termini di Abstandsprachen (ma solo uno dei due codici ha pieni requisiti di Ausbausprache: la varietà $\mathrm{B}$ non può esserlo perché non copre $\mathrm{i}$ domini alti e gli usi scritti formali) : ad es. la Svizzera tedesca, varie regioni italiane fino al tardo Ottocento (e i primi del Novecento) dove si notava infatti il rapporto di diglossia per eccellenza fra l'italiano e dialetti.

La dilalia differisce sostanzialmente dalla diglossia in quanto il codice A viene usato, almeno da una parte della comunità, anche nel parlato conversazionale quotidiano nonostante una chiara distinzione funzionale di ambiti di spettanza di A e di B rispettivamente : si notano impieghi e domini in cui di fatto si usano (e vengono considerati normali) sia l'una che l'altra varietà alternativamente 0 congiuntamente. Questa situazione, secondo BERRUTO (2005 : 207) è da ritenere tipica della maggior parte dell' area italo-romanza.

Infine, il bidialettismo o polidialettismo è la situazione in cui nel repertorio si avverte la presenza di una varietà standard e diverse varietà regionali e sociali, manca però un grado abbastanza alto di distanza tra diversi livelli di analisi : i parlanti padroneggiano con maggiore o minore sicurezza sia la varietà standard sia la varietà regionale e sociale, pur ricorrendo normalmente nella conversazione quotidiana solo a quest'ultima. Per la vicinanza strutturale dei due codici i parlanti non hanno una « reale coscienza di promozione di B come lingua alternativa » perciò sono frequenti degli usi commisti di A e B : ad es. la situazione in Inghilterra, in parte della Francia e, in Italia, è la situazione riscontrabile in Toscana e a Roma. BERRUTO (2005 : 209) specifica, a conclusione della sua trattazione, che diglossia dilalia e bidialettismo « si escludono a vicenda, ma neanche il bidialettismo è combinabile (considerando gli stessi codici o varietà di lingua) con nessuna delle altre tre situazioni. Lo è solo se sono diverse coppie di codici o varietà considerate ». Nella casistica presentata dallo studioso non si può avere allo stesso tempo bilinguismo sociale e diglossia (o dilalia, ecc.) mentre si rivela possibile la presenza di una specie di repertorio composto, in cui per es. a un bilinguismo sociale tra $\mathrm{X}$ e $\mathrm{Y}$ si sommi una diglossia o dilalia tra $\mathrm{X}$ e $\mathrm{Z}$ o tra $\mathrm{Y}$ e Z.

Secondo GRASSI (2001 : 254), che accetta il termine diglossia in riferimento alla prima metà del XX secolo, sarebbe più appropriato parlare di diglossia senza bilinguismo. Lo studioso fa notare che « in realtà, tutti conoscevano il dialetto, ma solo pochi dominavano anche l'italiano, cioè erano bilingui ». Lo conferma anche MiONI (1979: 108). Nel secondo Novecento, in seguito ai processi che favoreggiarono l'uso della lingua nazionale (urbanesimo, alfabetizzazione, 
migrazioni interne, sviluppo dei mezzi di comunicazione di massa, ecc.) in contrapposizione all'uso del dialetto e al passaggio a una società basata sull'industria, sul commercio, sulle comunicazioni, la situazione è radicalmente cambiata. GRASSI (1999 : 280) sostiene quindi che ai nostri tempi si tratti di diglossia con diversi gradi di bilinguismo e di bilinguismo con diglossia e sottolinea il passaggio graduale da « una prevalente diglossia a un prevalente bilinguismo ».

Secondo MiONI (1979 : 109), come abbiamo visto, la situazione italiana media (nelle regioni a un livello medio di sviluppo) andrebbe definita in termini di diglossia con bilinguismo sociale. Il linguista, esaminando casi particolari del quadro linguistico italiano, non esclude tuttavia fenomeni di bilinguismo sociale senza diglossia quando la maggioranza dei parlanti conosce sia l'italiano che il dialetto ma, a causa della notevole percentuale di immigrati provenienti da altre regioni, i dialetti essendo così diversi non si possono integrare nell'uso di tutta la comunità. È interessante inoltre rilevare la presenza delle zone di monolinguismo standard. In conclusione, MIONI (ibid.) constata : «dall'unificazione alla seconda guerra mondiale abbiamo un periodo di transizione da un monolinguismo dialettale quasi assoluto (con un numero limitato di parlanti bilingui) alla situazione attuale, la cui tendenza più avanzata è verso il monolinguismo italiano $»$.

$\mathrm{Al}$ termine delle nostre riflessioni riteniamo opportuno, per fare il punto sulla situazione italiana, riportare alcune considerazioni più particolareggiate sul caso italiano basandoci su una lista di criteri che permettono di analizzare meglio le situazioni di bilinguismo sociale, diglossia, dilalia o bidialettismo, stesa da BERRUTO (2005 : 205). In Italia, tranne che in Toscana e prevedibilmente a Roma (condizioni diverse), nella situazione media delle regioni italiane:

1. si riscontra la coesistenza negli usi di italiano e dialetto che nonostante l'affinità genetica:

a) presentano una sensibile diversità strutturale,

b) vengono entrambi usati nella conversazione ordinaria,

c) assumono funzioni nettamente diverse (alte l'italiano, basse il dialetto);

2. c'è la sovrapposizione di domini fra due codici (condividono classi di situazioni in cui le funzioni si sovrappongono);

3. il dialetto è poco o non affatto standardizzato;

4. il dialetto è socialmente marcato e stratificato in varietà sociali;

5. esiste un continuum di sottovarietà fra italiano e dialetto (l'italiano popolare e regionale molto marcato, il dialetto italianizzato ${ }^{37}$, ecc.);

6. l'italiano gode di alto prestigio;

7. sia l'italiano che (ancora) il dialetto sono presenti nella socializzazione primaria;

8. c'è la possibilità (o tentativi) di promozione del dialetto a codice alto;

9. è ricorrente l'impiego alternativo della lingua e del dialetto (sotto forma di commutazione di codice e di enunciazione mistilingue) nel parlato conversazionale;

\footnotetext{
${ }^{37}$ In : BERRUTO (1993 : 27-32).
} 
10. i dialetti (almeno alcuni di essi) sono portatori di una tradizione, e hanno un uso, letterario scritto, in poesia e in prosa.

Come possiamo constatare, dal quadro delineato sopra emerge chiaramente che la situazione linguistica italiana è tutt'altro che omogenea ed è praticamente impossibile definirla in maniera univoca, e quindi le condizioni di ogni regione andrebbero valutate separatamente. Lo conferma anche GRASSI (1964: 66) sostenendo che « non si dovrà più cercare di interpretare le situazioni locali partendo da dati generali validi per tutta la Nazione ». Tuttavia FREDDI (1983 : 232) conclude : «Oggi l'italianizzazione della penisola può dirsi sostanzialmente compiuta ».

Infatti, da un'indagine DoXA condotta nel 1988 (VIGNUZZI, $1988:$ 241) risultava comunque che circa il 34\% (di contro al 25\% del 1974) degli intervistati dichiarava di parlare italiano in famiglia, mentre il $23 \%$ ha permesso di concludere « quanto [fosse] salda la situazione di bilinguismo più o meno diglottico tra dialetto e lingua per larga parte della popolazione » (VIGNUZZI, 1988 : 244).

Mentre in base ai dati dell'ISTAT del 1993, si può già affermare seguendo RUSSO (1993 : 36) che «l'italofonia ha raggiunto la punta record dell' $85 \%$ », vale a dire : circa il $42 \%$ della popolazione dichiara di usare in modo esclusivo l'italiano in famiglia, circa il $45 \%$ quando parla con gli amici e attorno al $64 \%$ quando parla con estranei. Accanto agli italofoni esclusivi, ci sono parlanti che usano indifferentemente sia l'italiano sia il dialetto : sono il $25 \%$ negli scambi familiari, il $27,1 \%$ con gli amici e il $20,3 \%$ con gli estranei. Il dialetto è invece sicura lingua familiare per circa il $32 \%$, scende al circa $26,4 \%$ nelle conversazioni con gli amici e si riduce al 13,7\% con gli estranei. RUSSO (1993 : 36) conclude : « Come rileva anche l'ISTAT, il dialetto è dunque patrimonio noto e utilizzato da poco meno del $60 \%$ della popolazione e questo accade in concomitanza con il raggiungimento della notevole soglia dell'85\% di italofoni ». A queste considerazioni possiamo aggiungere che il repertorio linguistico italiano diventa sempre più articolato e conferma di evolvere non verso la sostituzione dello standard ai dialetti, ma per aggiunta dell'italiano alle preesistenti possibilità espressive dialettali, con il risultato di un arricchimento della « nostra realtà plurilingue » (RUSSO, $1993: 37)$. 


\section{BIBLIOGRAFIA}

BERRUTO, Gaetano (1977), Dialettologia e sociolinguistica in Italia, in SIMONE Raffaele, RUGGIERO Giulianella (a cura di), Aspetti sociolinguistici dell'Italia contemporanea, Roma, Bulzoni, pp. 77-86.

BERRUTO, Gaetano (1989), Tra italiano e dialetto, in Holtus Günter, METZELTIN Max (a cura di), La dialettologia italiana oggi. Studi offerti a Manlio Cortelazzo, Tübingen, Narr, pp. 107-122.

BERRUTO, Gaetano (1993), Le varietà del repertorio, in SOBRERO Alberto (a cura di), Introduzione all'italiano contemporaneo. Vol. I. La variazione e gli usi, Roma-Bari, Laterza, pp. 3-36.

BERRUTO, Gaetano (1993), Varietà diamesiche, diastratiche, diafasiche, in SOBRERO Alberto (a cura di), Introduzione all'italiano contemporaneo. Vol. I. La variazione e gli usi, Roma-Bari, Laterza, pp. 37-90.

BERRUTO, Gaetano (2003), Sociolinguistica dell'italiano contemporaneo, Roma, Carocci.

BERRUTO, Gaetano (2005), Fondamenti di sociolinguistica, Roma-Bari, Laterza.

CASTEllani Arrigo (1982) Quanti erano gli italofoni nel 1861 ?, Studi Linguistici Italiani 8/1, Roma, Salerno Editrice, pp. 3-26.

COVERI, Lorenzo, BENUCCI, Antonella (1998), Le varietà dell'italiano, Roma, Bonacci Editore.

FERGUSON, Charles A. (1964), Diglossia, in HyMes David (a cura di), Language in culture and society, London, Harper and Row, pp. 429-437.

FRANCESCATO, Giuseppe (1978), Dialetto, lingua regionale e lingua nazionale nella scuola, in LO CASCIO Vincenzo (a cura di), Prospettive sulla lingua madre, Roma, Istituto della Enciclopedia G. Treccani, pp. 127-149.

FREDDI, Giovanni (1983), L'Italia plurilingue, Bergamo, Minerva Italica

GRASSI, Corrado (1964), Comportamento linguistico e comportamento sociologico, Archivio Glottologico Italiano 49/1, pp. 40-66.

GRASSI, Corrado (1999), Italiano e dialetti, in SOBRERO Alberto (a cura di), Introduzione all'italiano contemporaneo. Vol. I. La variazione e gli usi, Roma-Bari, Laterza, pp. 279-308.

GRASSI, Corrado, SOBRERO, Alberto (2001), Fondamenti di dialettologia italiana, Roma-Bari, Laterza.

Hudson, Richard A. (1996), Sociolinguistica, Bologna, Il Mulino.

LOMBARDI SATRIANI, Luigi M. (1974), Dal dialetto alla lingua : riscatto culturale o perdita di identità ?, in AA.VV., Dal dialetto alla lingua, Pisa, Pacini Editore, pp. 91-100.

MionI, Alberto M. (1979), La situazione sociolinguistica italiana : lingua, dialetti, italiani regionali, in ColOMBo Adriano (a cura di), Guida all'educazione linguistica. Fini, modelli, pratica didattica, Bologna, Zanichelli, pp. 101-114.

RUSSo, Domenico (1993), Un aggiornamento sulle cifre di italofonia, Italiano e oltre 1, Firenze, La Nuova Italia, pp. 36-38.

Titone, Renzo (1973), Bilinguismo collettivo e dinamica degli scambi linguistici, Quaderni per la promozione del bilinguismo 2/12, pp. 1-20. 
TRUMPER, John (1977), Ricostruzione nell'Italia settentrionale : sistemi

consonantici. Considerazioni sociolinguistiche nella diacronia, in SIMONE

Raffaele, VignuZZI Ugo (a cura di), Problemi di ricostruzione in linguistica, Roma, Bulzoni, pp. 259-310.

VignuZZI, Ugo (1988), Chi parla ancora in dialetto ?, Italiano e oltre 5, Firenze, La Nuova Italia, pp. 241-245.

WEINREICH, Uriel (1974), Lingue in contatto, Torino, Boringhieri. 